\title{
Reine Frauenstationen in Psychiatrie und Psychotherapie
}

\author{
Special Wards for Women in Psychiatry and Psychotherapy
}

Autoren

Pro: Andrea Moldzio

Kontra: Tilman Steinert, Ralf-Peter Gebhardt

Bibliografie

DOI http://dx.doi.org/

10.1055/s-0030-1248587

Psychiat Prax 2010; 37:

319-321

(c) Georg Thieme Verlag KG

Stuttgart · New York .

ISSN 0303-4259

Korrespondenzadressen

Dr. med. Dr. phil. Andrea Moldzio, MBA

Asklepios Klinik Nord, Hamburg-Ochsenzoll

Langenhorner Chaussee 560

22419 Hamburg

a.moldzio@asklepios.com

\section{Prof. Dr. Tilman Steinert}

Zentrum für Psychiatrie Südwürttemberg, Abteilung Psychiatrie I der Universität Ulm 88190 Ravensburg

tilman.steinert@zfp-zentrum.de

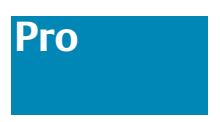

Entgegen zahlreicher Widerstände haben wir 2005 in der Asklepios Klinik Nord Ochsenzoll in Hamburg eine „Behandlungseinheit für Frauen“ (BeF), die nicht nur ausschließlich Frauen behandelt, sondern auch aus einem ausschließlich weiblichen Therapeutenteam mit Ärztinnen, Psychologinnen, Ergo- und Tanztherapeutinnen besteht, eröffnet. Behandelt werden schwerpunktmäßig Patientinnen mit Traumafolgestörungen (z.B. PTBS, dissoziative Störungen) sowie Frauen mit allgemeinpsychiatrischen Diagnosen, die den Wunsch haben, auf einer Frauenstation behandelt zu werden.

Sehr häufig hörten wir das Argument, dass reine Frauenstationen antiquiert wären, und letztlich nur einen Rückschritt in das „tiefe Mittelalter“ der Psychiatriegeschichte sind. In der Tat herrschte in der psychiatrischen Versorgung bis zur Mitte des 20. Jahrhunderts eine Geschlechtertrennung, welche männliche und weibliche Patienten weitgehend voneinander isolierte. Im Zuge der Humanisierung und Normalisierung der Psychiatrie, die sich in erster Linie gegen die restriktiven, deprivierenden und hospitalisierenden Krankenhauszustände wandte, setzte sich erst in den 50er-Jahren des 20. Jahrhunderts eine Mischung der Geschlechter auf den psychiatrischen Stationen durch, die durch die Psychiatrieenquete 1975 noch einmal ausdrücklich empfohlen wurde. Man erhoffte sich von der Geschlechtermischung in erster Linie eine weitgehende Normalisierung der therapeutischen Gemeinschaft der Patienten analog der Welt außerhalb der Klinik.

Der meistgeäußerte Kritikpunkt gegenüber unserer Behandlungseinheit für Frauen war, dass eine Trennung von Frauen und Männern eine künstliche Spaltung der Welt suggeriere. Dadurch würden Frauen mangels Kontakt oder Auseinandersetzung mit Männern die Hälfte der Realität ausblenden und sich kokonartig in eine irreale Schonwelt zurückziehen, die spätestens bei der unvermeidlichen Rückkehr in die Realität wie eine Seifenblase zerplatze.

Als weiteres Argument gegen eine Geschlechtertrennung wurde die möglicherweise verdeckte Implikation von Schuldzuweisungen an das männliche Geschlecht angeführt: Gerade bei einer Frauenstation mit dem Schwerpunkt der Traumatherapie läge die Gefahr darin, Frauen einseitig als Opfer und Männer als Täter zu stigmatisieren und damit dem Mann per se offen oder verdeckt die Täterrolle zuzuschreiben. Die Verfestigung eines einseitigen und problematischen Männerbildes wäre dann die Folge.

In Anbetracht des heutigen Wissensstandes greift jedoch die Frage, ob eine Frauenstation per se sinnvoll ist oder nicht, zu kurz. Eine differenziertere Frage wäre, unter welchen Bedingungen eine Behandlungseinheit für Frauen für welche Patientinnen sinnvoll ist.

In der gegenwärtigen Gesundheitsforschung ist eine geschlechtsdifferente Betrachtung von Erkrankungen ein zunehmend aktueller werdendes Thema. Frauenspezifische Fragestellungen werden nicht mehr nur im angloamerikanischen Raum, sondern zunehmend auch in Deutschland rezipiert und finden Eingang in gesundheitspolitische Konzepte [1-3]. Dennoch liegt die konkrete Versorgungsrealität psychiatrischer Patienten und Patientinnen weit hinter den aktuellen Forschungserkenntnissen über Geschlechterdifferenzen zurück, sodass geschlechtersensible Therapieangebote in der Psychiatrie bislang noch eine Rarität sind.

Besonders erstaunlich ist, dass es ausgerechnet für traumatisierte Patienten und Patientinnen kaum geschlechtsspezifische Angebote in der Psychiatrie gibt. Unterliegen doch gerade Gewalterfahrungen einer geschlechtsspezifischen Häufung und Überformung $[4,5]$. Vor allem unter psychiatrischen und psychosomatischen Patienten und Patientinnen in stationären Institutionen liegen allein die Prävalenzdaten von sexuellem Miss- 
brauch im Durchschnitt bei 20\% [6-8]. Wobei man aber davon ausgehen muss, dass in psychiatrischen Kliniken die tatsächliche Anzahl von Patienten mit einer Vorgeschichte mit sexuellem Missbrauch höher ist als überhaupt durch Studien erfassbar ist [9]. Zudem unterliegt die Krankheitsverarbeitung und -bewältigung geschlechtsspezifischen Prozessen: Beispielsweise entwickeln Frauen nach einem Trauma doppelt so häufig und lange eine posttraumatische Belastungsstörung als Männer [10].

Auch wenn die Normalität einer zweigeschlechtlichen Welt von unbestrittenem Wert ist, so ist die Möglichkeit der Wahlfreiheit und die Berücksichtigung der speziellen Bedürfnisse von Patienten und Patientinnen ein ebenso großer Wert, der in der psychiatrischen Versorgung etabliert sein sollte. Gerade für Patientinnen mit Gewalterfahrungen bieten „therapeutische Frauenschutzräume auf Zeit“ oft erst die Voraussetzung, sich auf eine Psychotherapie überhaupt einzulassen.

Die lebensgeschichtlich geprägte Vulnerabilität für Gewalt bei diesen Patientinnen bedarf als erstes eines sicheren, stressarmen, äußeren Rahmens ohne reale Bedrohungen, um ein Gefühl innerer Sicherheit entstehen zu lassen, auf dem alle weiteren therapeutischen Interventionen basieren.

Dem entgegen steht die Tatsache, dass es auf gemischtgeschlechtlichen Stationen, vor allem auf Akutstationen, durch männliche Patienten häufiger zu Bedrohungen, physischen und sexuellen Übergriffen gegenüber weiblichen Patientinnen kommt [11,12].

Auch Patientenbefragungen zur Folge beklagten sich v.a. weibliche Patienten über sexuelle Übergriffe und eine mangelnde Privat- und Intimsphäre auf gemischten Stationen, sodass studienübergreifend ca. 20-30\% aller Patientinnen getrenntgeschlechtliche Stationen bevorzugen würden [13].

Dies heißt jedoch keinesfalls, dass die Konfrontation mit anderen Frauen komplikationslos wäre, zumal auch häufig Mütter Täteroder Mittäterinnen sein können. Lediglich die konkrete Bedrohung im „Hier und Jetzt“ ist bei Frauen deutlich geringer und weniger sexualisiert $[14,15]$.

Von besonderer Bedeutung ist auch die Frage, was für spezielle Bedingungen auf einer solchen Frauenstation gegeben sein müssen, um geschlechtssensibel zu sein. Sicherlich ist es nicht mehr zeitgemäß, Frauen einfach nur von Männern zu separieren, ohne besondere geschlechtersensible Haltung oder spezielle therapeutische Ansätze.

Unserer Erfahrung nach ist es zusammenfassend sinnvoll, Patientinnen 1. mit einer störungsspezifischen Indikation (wobei die Posttraumatische Belastungsstörung hier nur exemplarisch ist) auf einer Frauenstation zu behandeln, wobei zur Indikation aber auch der Wunsch einer Patientin auf einer Frauenstation behandelt werden zu wollen, zählt und respektiert werden sollte. Und 2. sollte diese Frauenstation auch bestimmte Kriterien moderner Psychiatrie und Psychotherapie erfüllen, wie z.B. unter Einbezug des Wissensstandes über Frauengesundheit eine geschlechtersensible Haltung vertreten, frauenspezifische Themen in Zusammenhang mit soziokulturellen geschlechtsspezifischen Handlungsspielräumen in den Einzel- und Gruppentherapien reflektieren, adaptierte Psychopharmakotherapie für Frauen sowie methodenintegrierende und ressourcenorientierte Psychotherapie etc. anbieten. In diesem Rahmen gehört es auch dazu, ein reflektiertes und differenziertes Männerbild zu entwickeln, das feindseligen Übergeneralisierungen und verzerrenden Stigmatisierungen entgegenwirkt.

Wenngleich unsere Behandlungseinheit für Frauen nur ein Beispiel für die Verwirklichung einer geschlechtersensiblen Psychiatrie ist, die selbstverständlich auch für Männer anwendbar ist, so möchte ich dennoch die generelle Behauptung aufstellen, dass sich die Fortschrittlichkeit und Professionalität einer Klinik nicht zuletzt auch darin misst, wie störungsspezifisch und geschlechtssensibel sie ist.

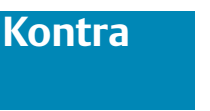
Die Frage der Geschlechtermischung in der Psychiatrie hat viel Geschichte. Viele Jahrzehnte vor der Durchmischung der Patienten drehten sich die Debatten um die Durchmischung des Personals. Auch dies galt seinerzeit als unvorstellbar. In der Klinik, in welcher wir heute arbeiten, erfolgte dies erzwungenermaßen 1914, als mit einem Schlag ein Großteil der männlichen „Wärter“ zum Kriegsdienst eingezogen wurde und man der Not folgend dazu übergehen musste, behelfsweise weibliches Personal auf den Männerstationen einzusetzen. Überraschenderweise konnte der Anstaltsdirektor danach konstatieren: „Die Verwendung von Wärterinnen auf der Männerabteilung hat sich ... aufs Beste bewährt. Die Sauberkeit der Abteilungen, der Ton unter Kranken und Personal sowie die Pflege der Kranken haben in gleicher Weise gewonnen. Irgendwelche Störungen oder Unannehmlichkeiten haben sich nicht ergeben“ [16]. 70 Jahre später erlebte ich (T.S.) zu Beginn meiner Weiterbildung als Assistenzarzt auf einer forensisch-psychiatrischen Langzeitstation die Aufregung, die entstand, als erstmals eine weibliche Patientin aufgenommen werden sollte und musste. Der Nachmittag ihres Eintreffens ist mir unvergesslich. Allen voran Detlef A., ein stets mit nacktem Oberkörper und langen ungepflegten Haaren auf der Station herumlungernder, zu keinerlei Aktivität irgendwelcher Art zu bewegender Patient. Pünktlich eine halbe Stunde vor dem Eintreffen der ersten Mitpatientin saß er lässig entspannt, frisch rasiert und frisiert im dunklen Anzug mit Krawatte im Aufenthaltsraum der Station. Die rasch eintretende Normalisierung und Gewöhnung beinhaltete (leider) auch, dass wenige Wochen später wieder alles beim Alten war. Kurzfristige (Honeymoon-)Effekte und längerfristige Alltagsrealität gilt es auch bei der empirischen Untersuchung dieses Themas zu berücksichtigen.

Als nächstes erlebten wir vor 20 Jahren - nahezu beschämend spät! - die geschlechtliche Mischung und Sektorisierung unserer allgemeinpsychiatrischen Aufnahmestationen. Die Dramatik spielte sich in den monatelangen vorangehenden Diskussionen ab. Bis dahin war die Männerstation ein finsteres Ambiente, in dem man sich, als diensthabender Arzt hinzugerufen, intuitiv eher mit dem Rücken an der Wand orientierte. In der Frauenstation dagegen war das Bild stets von mehreren agitierten Patientinnen geprägt, die jeden hinzukommenden Arzt sofort nach Öffnen der Glastüre mit ihren unterschiedlichsten Anliegen bestürmten und bedrängten. Diese beiden Milieus mischen? Schwerste Bedenken wurden ins Feld geführt. Zum Beispiel das Männerproblem. Die bisherige Frauenstation hatte scheinbar zu wenig männliche Mitarbeiter. War es zu verantworten, eine Station mit männlichen psychotischen Patienten zu führen, ohne dass mindestens 2 kräftige Männer gleichzeitig im Dienst waren? Oder das Frauenproblem. Hier wurde nämlich seitens der bisherigen Frauenstation die „Spezialkompetenz für die Behandlung psychosekranker Frauen“ reklamiert. Unsicherheit machte sich auch auf der Männerstation breit. Welche unwägbaren Komplikationen waren zu gewärtigen, wenn eine Patientin ihre Periode bekommt? Müssten womöglich gar Schwangere behandelt werden? Wie überall, wo dieser Prozess vollzogen wurde, lösten sich alle diese Bedenken nach der erfolgten Durchmischung binnen weniger Wochen auf. Aufgrund der vielfältigen Befürchtun- 
gen und kontroversen Diskussionen im Vorfeld dieses die Klinik sehr bewegenden Vorhabens wurde eine Begleitforschung mit dem erklärten Ziel entwickelt, mögliche unerwünschte Nebenwirkungen der geschlechtlichen Mischung empirisch zu erfassen [17-19].

Vier akutpsychiatrische Stationen wurden 7 Monate vor, 2 Monate nach und nochmals 11 Monate nach der Geschlechtermischung untersucht. Über die 3 Messzeitpunkte befanden sich jeweils andere Patienten in Behandlung, während das Personal nur geringfügig fluktuierte. Von den insgesamt 239 Patienten konnten 77\% (101 Frauen, 82 Männer) befragt werden. Beim Personal betrug der Rücklauf 61 \% (101 Frauen, 61 Männer). Patienten und Personal beurteilten die Stationsatmosphäre mit dem Stationsbeurteilungsbogen (SBB) und wurden nach ihrer persönlichen Einstellung zur geschlechtlichen Mischung befragt. Ein weiterer zentraler Fragenkomplex an Patienten und Personal war, ob sie in der vergangenen Woche vor der Befragung verbale oder körperliche Bedrohungen oder verbale oder körperliche sexuelle Belästigungen oder Übergriffe zwischen den Patienten oder zwischen Patienten und Personal gesehen oder erlebt hätten. Die wesentlichen Ergebnisse waren:

- Nach der geschlechtlichen Mischung wurde die Stationsatmosphäre zu beiden Messzeitpunkten vonseiten des Personals als signifikant und von den Patientinnen und Patienten als leicht verbessert eingeschätzt.

- Unisono berichteten Personal, Patientinnen und Patienten zu beiden Messzeitpunkten nach der geschlechtlichen Mischung von hochsignifikant weniger aggressiven Vorkommnissen als vor der geschlechtlichen Mischung.

- Während 2 Monate nach der geschlechtlichen Mischung zunächst weniger sexuelle Belästigungen als vor der geschlechtlichen Mischung beobachtet worden waren, wurden 11 Monate nach der geschlechtlichen Mischung signifikant mehr sexuelle Belästigungen zwischen den Patienten und zwischen Patienten und Personal beobachtet als vor der Mischung.

- Die geschlechtliche Mischung war im Vorfeld von 66\% des Personals gewünscht worden, nach der geschlechtlichen Mischung lag die Zustimmungsquote des Personals bei $94 \%$.

- Vor der geschlechtlichen Mischung sagten 56\% der Patienten, dass sie eine geschlechtlich gemischte Station präferieren würden. Danach waren fast $92 \%$ der Patienten für die geschlechtliche Mischung (99\% der Männer, 83\% der Frauen).

Die Ergebnisse zeigen, dass die geschlechtliche Durchmischung im Durchschnitt sowohl nach unseren Daten als auch nach den verbreiteten klinischen Erfahrungen für die Patienten und für den Betrieb einer psychiatrischen Klinik sinnvoll ist. Insbesondere hatte die Geschlechtermischung eine nachhaltige Reduktion aggressiver Vorkommnisse zur Folge. Die Ergebnisse bilden aber, wie in derartigen Untersuchungen üblich, einen statistischen Durchschnitt ab und lassen keine Rückschlüsse für den Einzelfall zu. Unbestreitbar kommt es nicht häufig, aber doch ab und zu vor, dass Patientinnen in der einen oder anderen Weise vor Mitpatienten geschützt werden müssen und dass dies nicht immer optimal gelingt. Dass dies in unserer Gesellschaft auch so ist, ist kein ausreichendes Argument, schließlich hat eine Klinik auch Schutz zu bieten. Es sei deshalb in dieser Frage auf eine weitere Erkenntnis der Evidenzklasse IV - schlichte persönliche Erfahrung - verwiesen.

Auf unserer Kriseninterventionsstation, auf der vorwiegend (aber eben nicht ausschließlich) weibliche Borderline-Patientinnen behandelt werden, viele davon mit einer ausgeprägt traumatisierten Vorgeschichte, kommt es immer wieder einmal dazu, dass wir Patientinnen mit einer Opfergeschichte zusammen mit männlichen
Patienten mit einer Tätergeschichte behandeln müssen. Die Brisanz ist aber auch hier geringer als erwartet. Die männlichen Patienten passen sich in ihrem Auftreten an und nehmen sich zurück oder sie geraten so stark unter Druck von Mitpatientinnen und Mitarbeiterinnen, dass sie die Behandlung abbrechen. Nicht optimal, aber für die betroffenen Frauen meistens eine wirklichkeitsnahe Situation, in der sie sich mit Unterstützung zu behaupten lernen. Zu tatsächlichen tätlichen Übergriffen ist es dort noch nie gekommen. Dies scheint uns auch als generelle Richtung tauglich: Künstliche, der Wirklichkeit nicht entsprechende Schonräume können auch therapeutisch schnell mehr Nachteile als Vorteile bringen. Wohl aber bedarf es einer Stationskultur, in der eine hohe Sensibilität für die Rechte von Frauen herrscht und alle Mitarbeiterinnen und Mitarbeiter stets und unzweideutig für diese eintreten. Eine sinnvolle Ergänzung können „Frauenzonen“ auf einer Station sein, zu denen die männlichen Mitpatienten keinen Zutritt haben, sofern die Architektur solche Lösungen zulässt.

\section{Literatur}

1 Hurrelmann K, Kolip P. Geschlecht, Gesundheit und Krankheit. Bern: Huber, 2001

2 Riecher-Rössler A, Bitzer J. Frauengesundheit. München, Jena: Urban und Fischer, 2005

3 Rohde A, Riecher-Rössler A. Psychische Erkrankungen bei Frauen. Freiburg: Karger, 2001

4 Kessler RC, Sonnega A, Bromet E et al. Posttraumatic stress disorder in the National Comorbidity Survey. Archives of General Psychiatry 1995; 52: $1048-1060$

5 Resnick HS, Kilpatrick FG, Dansky BS et al. Prevalence of civilian trauma and posttraumatic stress disorder in a representative national sample of women. Journal of Consulting and Clinical Psychology 1993; 61: 984-991

6 Cloitre M, Tardiff K, Marzuk P et al. Childhood Abuse and Subsequent Sexual Assault Among Female Inpatients. Journal of Traumatic Stress 1996; 9: 473-482

7 Convoy H, Weiss P, Zverina J. Sexual abuse experiences of psychiatric patients. Med Law 1995; 14: 283-292

8 Ellerbrok G, Heuft G, Senf W. Zur Prävalenz sexuellen Missbrauchs in der Vorgeschichte stationärer Psychotherapiepatienten. Der Psychotherapeut 1995; 40: 9-16

9 Richter-Appelt H, Moldzio A. Psychotherapie mit Patientinnen nach sexueller Traumatisierung. In: Körner W, Lenz A, Hrsg. Sexueller Missbrauch. Band 1: Grundlagen und Konzepte. Göttingen: Hogrefe, 2004: 413-432

10 Breslau N. Sex differences in posttraumatic stress disorder. Archives of General Psychiatry 1997; 54: 1044-1048

11 Thomas C, Barlett A, Mezey GC. The extent and effects of violence among psychiatric inpatients. Psychiatric Bulletin 1995; 19: 600-604

12 Vieweg T, Schubert R, Lemke S. Das Stationsklima gemischt- und getrenntgeschlechtlicher akutpsychiatrischer Stationen. Psychiat Prax 1996; 23: 290-293

13 Spießl H, Frick U, Kovatsits $U$ et al. Getrennt- oder gemischt-geschlechtliche Behandlung in der psychiatrischen Klinik aus Sicht der Patienten. Nervenarzt 2001; 72: 515- 520

14 Cleary $M$, Warren R. An exploratory investigation into women's experiences in a mixed sex psychiatric admission unit. Australian and New Zealand Journal of Mental Health Nursing 1998; 7: 33-40

15 Mezey G, Hassell Y, Barlett A. Safety of women in mixed-sex and singlesex medium secure units: staff and patient perceptions. British Journal of Psychiatry 2005; 187: 579- 582

16 Steinert T. Die Geschichte des psychiatrischen Landeskrankenhauses Weissenau. Darstellung der Anstaltsgeschichte von 1888 bis 1945 im ideengeschichtlichen und sozio-ökonomischen Kontext. Weinsberg: Weißenhof, 1984: 59

17 Gebhardt RP, Steinert T. Schwierige Patienten konzentrieren oder verteilen? Auswirkungen von innerer Sektorisierung, partieller Stationsöffnung und Geschlechtermischung auf das Behandlungsmilieu. Psychiat Prax 1999; 26: 61-66

18 Gebhardt RP, Steinert T. Should severely disturbed patients be distributed or concentrated in specialized wards? An empirical study on the effects of hospital organization on ward atmosphere, aggressive behavior, and sexual molestation. Eur Psychiatry 1999; 14: 291-297

19 Gebhardt RP, Steinert T. Einstellungen von Patienten und Personal zu gemischtgeschlechtlichen Stationen in der Akutpsychiatrie. Krankenhauspsychiatrie 2000; $11: 21-24$ 